\title{
Allergy and high trait anxiety are related to increases in heart rate variability: results of naturalistic long-term design study
}

\author{
Department of Psychology, Faculty of Arts, Comenius University, Gondova 2, 81499 Bratislava, Slovakia
}

\section{KEY WORDS}

heart rate variability; allergy; trait anxiety; stress; naturalistic design

\section{Corresponding author}

Jakub Rajcani

Gondova 2

81499 Bratislava

Slovakia

E-mail: jakub.rajcani@uniba.sk

\section{Doi}

10.23822/EurAnnACI.1764-1489.33

\begin{abstract}
Summary
A number of studies report heart rate variability (HRV) changes in allergic as well as high trait anxious people, and associations between allergic inflammation and trait anxiety. This study investigated HRV of 20 low anxious allergic, 19 healthy high trait anxious and 18 healthy low anxious, in naturalistic setting. On arranged research days, subjects performed measurements using portable ECG device and subjective self-assessment of perceived stress. Five repeated measurements data from each subject have shown increases in overall HRV, as well as HRV on respiratory frequencies in both allergy and high trait anxiety. Subject's sex was an important factor, because HRV increases in allergy were only apparent in women. Data from self-assessment show no differences in experienced stress attributable to allergy, only to trait anxiety.
\end{abstract}

\begin{abstract}
Abbreviations
ANS, autonomic nervous system; HR, heart rate; HRV, heart rate variability; IgE, immunoglobulin $\mathrm{E}$; $\mathrm{AR}$, allergic rhinitis; $\mathrm{AD}$, atopic dermatitis; BMI, body mass index; STAI, state and trait anxiety inventory; ECG, electrocardiogram; PSS, perceived stress scale; DFA, detrended fluctuation analysis; HF, high frequencies; LF, low frequencies; VLF, very low frequencies; ApEn, approximate entropy; BIC, Schwarz's Bayesian Criterion; F, test statistic; df, degrees of freedom.
\end{abstract}

\section{Introduction}

An increasing number of studies show relevance of autonomic nervous system (ANS) dysregulations to both mental and somatic disorders, mainly those which are related to stress (1). Autonomic dysregulations, may emerge as consequences of allostatic load, cumulative wear and tear of bodily systems, that have been exposed to chronic or traumatic stress $(2,3)$.
Heart rate (HR) is conditioned by both autonomic branches; normally it should exhibit a relatively large inter-beat variability. Heart rate variability (HRV) is used as a marker of healthy adaptive capacity of cardiovascular system, while cardiac pathological states show low or almost no variability and high predictability of heart rate $(4,5)$. Psychobiological models of neurovisceral integration $(6,7)$ underline associations of HRV with brain pathways connected to stress, therefore HRV may serve as a suitable psychobiological index of central inhibitory control and emotional regulation.

Anxiety is defined by Spielberger (8) as both an unpleasant emotional state grounded in fear (state anxiety), and as a relatively stable tendency (trait anxiety) to manifest anxiety states. High trait anxiety is related to failure in inhibition of threat responses on psychological as well as physiological level (9), which leads to perpetuation of the adaptive capacity of an individual. 
High anxiety and anxiety disorders were previously associated with increased cardiovascular risk (10), reduced overall HRV, and vagal HRV indices in anxiety disorders were concluded in recent meta-analysis (11). While findings in pathological anxiety are quite consistent across various studies (for review, see 11), HRV changes in subclinical anxiety are not so univocal. The assumption that chronic anxiety may be associated with trait-like lowered vagal tone (9) was supported by some studies, which found lowered HRV in highly anxious people $(12,13)$. However, there has been a problem with replication of these results on other data, with small effects attributable to anxiety, while acute stress related reductions of HRV were reliably observed (14).

\section{Anxiety and allergy}

Current neuroendocrinology research on allergy underlines the importance of psychological factors, such as stress, in the onset and pathogenesis of allergies (15). Cumulative allostatic load may play a part in allergic inflammatory process as well as changes in psychological states, e.g. feelings of anxiety (3). Increased trait anxiety was correlated with elevated levels of $\operatorname{IgE}$ and increase in Th2 immune response, which play a critical role in development of allergy (16). Other studies $(17,18)$ found that personality traits such as anxiety, depression, a high emotional excitability, as well as higher comorbidity of anxiety disorders were linked to major allergic disorders (atopic dermatitis, allergic rhinitis, allergic asthma).

Based on associations between allergy and anxiety, as well as on expected discomfort due to the presence of allergic symptoms, reduced HRV in allergy would be expected. Recent HRV studies, however, show that allergic patients exhibited an increase in overall HRV, as well as in HRV on respiratory frequencies. Increased HRV in adults suffering from allergic rhinitis (AR) $(19,20)$ as well as children $(21)$, imply parasympathetic predominance and/or sympathetic withdrawal in AR. Similar findings were obtained for atopic dermatitis (AD) as well (22).

Based on described links between high trait anxiety and allergy, we aim to investigate HR, HRV and subjectively perceived stress differences, in allergic and high trait anxious people. Because of strong comorbidity of allergy and high trait anxiety, it is difficult to access effects of allergy and anxiety on psychological and physiological functioning separately (23). We studied differences between groups of low anxious allergic, and high anxious healthy subjects, and compared them to low anxious healthy controls, to examine factors of allergy and high trait anxiety independently.

Another important characteristic of this study is the naturalistic design of data acquisition. Following our previous studies with laboratory design (24), and based on naturalistic studies with salivary cortisol (25) and studies of long term HRV or HRV in natural conditions $(19,26)$, we investigated HRV indices and subjective experience of research subjects in relation to stressors during their daily lives. A design with repeated measurements of HRV and subjective self-assessment of experienced stress during longer time period was applied here.

\section{Method}

\section{Subjects}

Subjects were recruited from students of Comenius University, Bratislava, while some allergy subjects were recruited from ambulant allergologists in Bratislava, Slovakia. Initially 64 people were recruited, however due to drop out of 7 of them the sample for analysis consisted of 57 subjects (26 males, 31 females) divided into three balanced groups based on allergy and anxiety. Assignment to allergy group was assessed based on individual history (occurrence of allergic symptoms for at least 2 years) and diagnosis of allergy based on a skin prick test performed by an allergologist. Both subjects with allergic rhinitis (AR) and atopic dermatitis (AD), as well as combined symptoms of both, were included. Subjects in non-allergy groups had no allergy symptoms and negative allergy anamnesis dating back to childhood. Trait anxiety was measured via Slovak version of State and trait anxiety inventory (STAI-T) $(11,27)$. Subjects were allocated to high and low anxiety groups based on cut off score: low anxiety $<39$, high anxiety $>49$. All who scored in between these values were not included in this study. This design was chosen based on prior stress research on Slovak sample (28) to include only very anxious, and on the other hand low anxious people.

Subjects suffering from cardiovascular, endocrine, psychiatric or any other chronic disease, which could affect HR and HRV variables, and subjects who regularly took medication (e.g. antihistamines, corticosteroids for allergy), or had any other possibly confounding conditions were excluded from the study. Some included allergic subjects took allergic medication seasonally, therefore only measurements from medication-free periods were included in data analysis.

Written informed consent was obtained from all subjects after explanation of all research procedures. The study was approved by ethics committee of the Trnava Self-Governing Region, $\mathrm{Tr}$ nava, Slovakia.

\section{Data Collection}

This study used repeated measurements design, with HR and HRV data collected between November 2014 and November 2016 in separate measurements. Research subjects were given randomly selected dates, during their everyday lives, in which data collection was performed. Moreover, one stressful and one 
relax day were individually agreed upon with each subject, relative to events in subjects' lives. This study includes data from 5 measurements from each subject, including both one stressful and one relax day.

ECG recording was performed via portable eMotion Faros $90^{\circ}$ or Faros $180^{\circ}$ ECG devices. Electrodes of ECG device were attached to subject's chest - one on the right side, under the clavicle, the other on the left under the ribcage.

At the beginning of data collection, all subjects were given instructions on how to apply the portable device and how to start and terminate the recording. All information was given to subjects in spoken as well as written form, and on research website (www.dlhodobymonitoring.sk), where they could also find dates of measurements, and all necessary information. Subjects could also contact research team via telephone, and were also regularly informed on research procedures.

Twenty-min ECG recordings and subjective emotional assessments were done in the morning, afternoon and evening, corresponding to sample collection for salivary cortisol, which will be analyzed and published separately. During all 20-min ECG measurements, subjects were instructed to be seated and relaxed. Evening ECG measurements were chosen as most appropriate for HRV analysis, due to the least missing data and movement artifacts. Morning and afternoon measurements were investigated via the same analyses and yielded congruent results to those described here.

Subjective assessment checklist was presented in paper form with separate pages for each measurement, containing questions about possible confound variables (e.g. alcohol, smoking, active movement or activity before measurement) and emotional states via list of adjectives compiled for this study. Used adjectives were related to feelings of stress, exhaustion and positive affect, which were proposed as latent factors via exploratory factor analysis.

For assessment of more long-term perception of stress, subjects filled Slovak version of perceived stress scale (PSS) (29) on each research day. PSS scale contains 10 statements that assess the extent in which a subject perceives their life as unpredictable, uncontrollable and overloading. Moreover, subjects were also instructed to write down stressful events which influenced them on the measurement day, and rate their stressfulness on a 5-point scale.

\section{Heart rate variability analysis and measures}

HR and HRV measures were obtained from ECG recording with sampling rate $250 \mathrm{~Hz}$. For purposes of HRV, two 5-min long windows were extracted from each recording and averaged. HRV analysis was realized via Kubios HRV software (30), and all included R-R intervals were manually checked for artifacts and ectopic beats. Only continuous 5 -min artifact free periods were included. Deleted ectopic beats represented less than $1 \%$ of used R-R intervals. Subsequently, data were analyzed using time domain parameters, spectral analysis via fast Fourier transform and non-linear analyses of Poincare plot characteristics, and fractal characteristics such as Entropy and Detrended fluctuation analysis (DFA).

As indicators of overall HR variability, we used SDNN (standard deviation of all R-R intervals) from time-domain analysis, Total power from spectral analysis, and SD2 (standard deviation of long term RR interval variability) from Poincaré plot. All these parameters are normally highly correlated.

Similarly, HRV on respiratory frequencies, which are predominantly associated with vagal activity, were used as follows: RMS$\mathrm{SD}$ (the square root of mean squared successive R-R differences) from time domain analysis, high frequency (HF) HRV fluctuations (frequency: $0.15 \sim 0.4 \mathrm{~Hz}$ ) from spectral analysis, and SD1 (standard deviation of short term RR interval variability) from Poincaré plot. These indices capture fast beat-to beat changes on respiratory frequencies which are attributable to inhibitory control via vagal nerve $(5,7,31)$. We also investigated HRV on lower frequencies $(\mathrm{LF})(0.04-0.15 \mathrm{~Hz})$ and very low frequencies (VLF) $(<0.04)$ that are thought to be modulated by a mix of both sympathetic and vagal influences as well as baroreflex influences, thermoregulatory processess etc. $(31,32)$.

From non-linear HRV parameters, we analyzed approximate entropy (ApEn) as a measure of overall complexity and unpredictability of HR series (33) and Detrended fluctuation analysis (DFA). DFA is a scaling method, which estimates fractal (self-similarity) attributes on both short term $\alpha 1$ (4-11 heart beats) and long term $\alpha 2$ scale $(33,34)$.

\section{Statistical analysis}

HRV measures naturally show large variances between measures. To enable statistical analyses, HRV variables from time domain, spectral and Poincaré plot analysis (SDNN, RMSSD, Total power, VLF, LF, HF, SD1 and SD2) were transformed by $\log 10$. Non-linear HRV variables (ApEn, DFA 1 1, DFA 2 ) were normally distributed, therefore remained untransformed. To investigate both between and within subject effects, mixed regression modeling was used. This approach is appropriate for multiple repeated measures with both fixed and random parameters (35). Analysis was performed in IBM SPSS 20 software. Mixed regression modeling used HR and HRV, and subjectively perceived stress measures as outcome variables in separate subsequent analyses. Categories of sex and research groups were used as fixed factors, while subject identity during five repeated measurements was a random factor. Unstructured co-variance structure was used due to the number of repeated measurements and unknown co-variance structure of the data. Further reported model provided the best fit while comparing Schwarz's Bayesian Criterion (BIC) to models with different combination of factors or co-variation structures. 
Table 1 - General characteristics of research sample (body mass index and age - mean + std. error).

\begin{tabular}{llcccc}
\hline & N & \multicolumn{2}{c}{ BMI } & \multicolumn{2}{c}{ Age } \\
\hline a, allergy + low trait anxiety & 20 subjects (10 males, 10 females) & 23.15 & \pm 0.679 & 21.3 & \pm 0.805 \\
\hline b, healthy + high trait anxiety & 19 subjects (8 males, 11 females) & 20.679 & \pm 0.481 & 20.421 & \pm 0.618 \\
\hline c, healthy + low trait anxiety & 18 subjects (8 males, 10 females) & 22.422 & \pm 0.602 & 19.611 & \pm 0.231 \\
\hline
\end{tabular}

Furthermore, relationships between HRV measures and subjective stress measures were investigated via partial correlations controlled for base heart rate of the subject. This was done to control for large interindividual variability in basal HR.

\section{Results}

The sample analyzed in this study consisted of 57 subjects (26 males, 31 females). To compare allergy and non-allergy highly anxious subjects, three research groups were formed: a, Allergic subjects with low trait anxiety $(n=20)$; b, highly trait anxious subjects with no allergy symptoms $(\mathrm{n}=19)$ and $\mathrm{c}$, low anxious controls with no allergy symptoms $(\mathrm{n}=18)$. All groups were balanced based on subjects' sex, age and body mass index (BMI) (for a summary of groups, see Table I).

\section{Assessing effects of allergy and anxiety}

Results of multiple regression model for individual outcome variables showed fixed effect of research group on overall HRV: SDNN: $\mathrm{F}(\mathrm{df}=2,55.92)=6.05, \mathrm{p}<0.01$; Total spectral power: $\mathrm{F}(2,55.18)=4.21, \mathrm{p}<0.02$; SD2: $\mathrm{F}(2,58.29)=6.49, \mathrm{p}<$ 0.01 . A similar effect was observed in HRV on respiratory frequencies: RMSSD: $\mathrm{F}(2,58.83)=3.82, \mathrm{p}<0.03$; HF-HRV: $\mathrm{F}$ $(2,56.94)=3.53, \mathrm{p}<0.04$; SD1: $\mathrm{F}(2,58.88)=3.6, \mathrm{p}<0.04$. Separate comparison of allergy and high trait anxiety group with controls is shown in following Table II, which summarizes mean values for individual variables and estimated difference of allergy and anxiety groups from controls.

HRV indices from spectral analysis, which represent slower changes in HR, have shown significant between group differences in VLF: $\mathrm{F}(2,51.2)=4.63, \mathrm{p}<0.02$, but not LF F (2, $56.42)=1.48, \mathrm{p}=0.24$.

Differences in non-linear variables for allergy and trait anxiety were not significant: ApEn: $F(2,57.06)=0.36, p=0.7$; DFA parameter $\alpha 1$ : F $(2,58.81)=0.83, \mathrm{p}=0.44$; DFA parameter $\alpha 2$ $\mathrm{F}(2,58.96)=1.25, \mathrm{p}=0.3$. Although no statistically significant differences were observed in non-linear parameters, $\alpha 1$ showed opposite trend in allergy and trait anxiety group to the rest of HRV variables in time domain and spectral analysis (see Table II). Effects of research group on basal heart rate (in beats per minute, bpm) was not significant $F(2,56.28)=2.31, p=0.11$, however difference between allergy and control group was significant (see Table II).

\section{Assessing effects of sex}

Differences between men and women in HR and HRV were assessed as a fixed factor. Women showed significantly higher heart rate $(80.856 \mathrm{bpm})$ than men $(74.014 \mathrm{bpm}) \mathrm{F}(1,58.35)$ $=13.41, \mathrm{p}<0.01$. Sex was also a statistically significant factor for overall HRV: SDNN: F $(1,58.07)=4.64, \mathrm{p}<0.04$; total spectral power: $\mathrm{F}(1,57.34)=7.18, \mathrm{p}<0.01$; SD2: $\mathrm{F}(1,60.34)$ $=6.68, \mathrm{p}<0.01$. Mean values for men and women as well as estimated differences are summarized in Table III.

Table II - Between group differences in HR and HRV.

\begin{tabular}{lccc}
\hline \multicolumn{1}{c}{$\begin{array}{c}\text { HR } \\
\text { and HRV } \\
\text { variables }\end{array}$} & allergy & $\begin{array}{c}\text { Experimental group } \\
\text { high trait } \\
\text { anxiety }\end{array}$ & control \\
\cline { 2 - 4 } HR $(b p m)$ & $75.355(-4.775)^{1}$ & $76.819(-3.311)$ & 80.13 \\
\hline $\begin{array}{l}\text { SDNN } \\
(\log 10)\end{array}$ & $1.838(+0.119)^{2}$ & $1.830(+0.111)^{1}$ & 1.719 \\
\hline $\begin{array}{l}\text { RMSSD } \\
(\log 10)\end{array}$ & $1.651(+0.152)^{1}$ & $1.648(+0.149)^{1}$ & 1.499 \\
\hline TP $(\log 10)$ & $3.622(+0.194)^{1}$ & $3.611(+0.183)^{1}$ & 3.428 \\
\hline $\begin{array}{l}\text { VLF } \\
(\log 10)\end{array}$ & $3.230(+0.213)^{2}$ & $3.212(+0.195)^{1}$ & 3.017 \\
\hline LF $(\log 10)$ & $3.154(+0.123)$ & $3.119(+0.088)$ & 3.031 \\
\hline HF $(\log 10)$ & $2.812(+0.269)^{1}$ & $2.797(+0.254)^{1}$ & 2.543 \\
\hline $\begin{array}{l}\text { SD1 } \\
(\log 10)\end{array}$ & $1.501(+0.148)^{1}$ & $1.499(+0.146)^{1}$ & 1.353 \\
\hline $\begin{array}{l}\text { SD2 } \\
(\log 10)\end{array}$ & $1.958(+0.116)^{2}$ & $1.949(+0.106)^{2}$ & 1.842 \\
\hline ApEn & $1.113(+0.012)$ & $1.111(+0.009)$ & 1.101 \\
\hline DFA 1 & $1.194(-0.069)$ & $1.212(-0.05)$ & 1.263 \\
\hline DFA 22 & $0.852(+0.001)$ & $0.889(+0.038)$ & 0.852 \\
\hline SF & & & \\
\hline
\end{tabular}

Estimated marginal means values, parameter estimate values compared to control group in brackets. Statistical significance value $(\mathrm{p}):{ }^{1}<0.05,{ }^{2}<0.01$. 
Table III - Sex differences in HR and HRV.

\begin{tabular}{lccc}
\hline \multirow{2}{*}{$\begin{array}{c}\text { HR and HRV } \\
\text { variables }\end{array}$} & \multicolumn{2}{c}{ Sex } & $\begin{array}{c}\text { Parameter } \\
\text { estimate }\end{array}$ \\
\cline { 2 - 3 } HR $(\mathrm{bpm})$ & female & male & $+6.841^{2}$ \\
\hline SDNN $(\log 10)$ & 1.763 & 1.829 & $-0.067^{1}$ \\
\hline RMSSD $(\log 10)$ & 1.570 & 1.629 & -0.059 \\
\hline TP $(\log 10)$ & 3.472 & 3.635 & $-0.164^{2}$ \\
\hline VLF $(\log 10)$ & 3.073 & 3.233 & $-0.160^{1}$ \\
\hline LF $(\log 10)$ & 3.030 & 3.173 & $-0.143^{1}$ \\
\hline HF $(\log 10)$ & 2.708 & 2.727 & -0.019 \\
\hline SD1 $(\log 10)$ & 1.423 & 1.479 & -0.056 \\
\hline SD2 $(\log 10)$ & 1.879 & 1.954 & $-0.075^{1}$ \\
\hline ApEn & 1.124 & 1.092 & $+0.032^{1}$ \\
\hline DFA $\alpha 1$ & 1.174 & 1.272 & $-0.098^{1}$ \\
\hline DFA $\alpha 2$ & 0.854 & 0.875 & -0.021 \\
\hline
\end{tabular}

Estimated marginal means values for men and women and parameter estimate values. Statistical significance value $(\mathrm{p}):{ }^{1}<0.05,{ }^{2}<0.01$.
Respiratory frequencies HRV have shown no significant main effect of sex. However, the interaction of both research group and sex was observed for HF-HRV F $(2,50.76)=4.41, \mathrm{p}<$ 0.02 and similar not significant interaction for RMSSD: $\mathrm{F}(2$, $50.97)=2.75, \mathrm{p}<0.07$. Different results in allergy and anxiety comparison between men and women were observed in other HRV variables as well; the interaction of factors was significant only in respiratory HRV parameters. Comparison of men and women in three research groups is shown in Figure 1.

Differences between sexes were also observed in non-linear HRV parameters of entropy (ApEn) F $(1,58.01)=6.95$, p < 0.01 , which was higher in women; and detrended fluctuations analysis $\alpha 1 \mathrm{~F}(1,60.52)=4.65, \mathrm{p}<0.04$, higher in men (for summary see Table III).

\section{Subjectively perceived stress differences}

For assessing subjectively perceived stress, Slovak translation of perceived stress scale (PSS) (Cohen, Kamarck, and Mermelstein, 1983) (29), and assessment of adjectives describing emotional states related to stress, exhaustion and positive affect, were used.

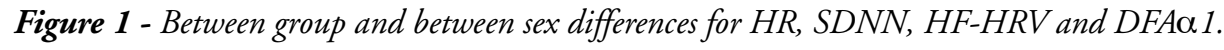
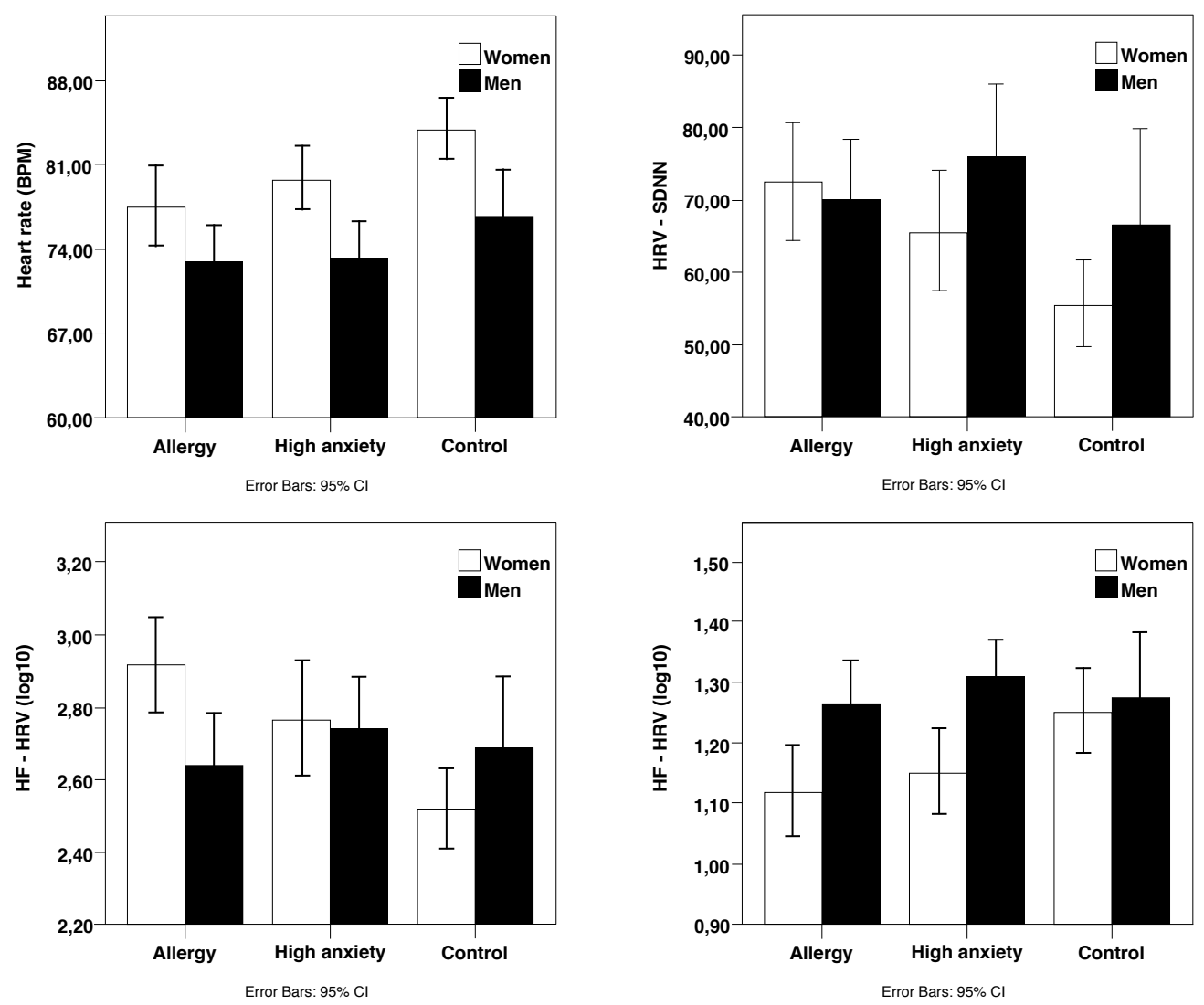
In multilevel regression model with the same factors as $\mathrm{HR}$ and HRV analysis, score from PSS differed significantly $(\mathrm{p}<0.01)$ between high trait anxiety group (mean score 21.51) and two remaining groups (allergy 16.00; control 14.93). Differences between sexes were not significant $(\mathrm{p}=0.12)$, women however showed slightly higher mean values (18.31) than men (16.66). Group differences between scores from adjective scales were found for stress adjectives (non-parametric Kruskal-Wallis test, $\mathrm{p}<0.01$ ), with higher score in high trait anxiety group (10.29) compared to allergy (8.38) and control group (8.02), and positive emotion adjectives $(\mathrm{p}<0.01)$ in high trait anxiety group (9.83), allergy (11.88), control (11.92). No statistically significant differences were found in exhaustion adjectives.

The relationship between HRV and subjective measures was investigated using partial correlations controlled for variable of heart rate, to eliminate individual differences in basal HR. Perceived stress (raw score from PSS test) correlated positively with VLF-HRV $(\mathrm{r}=0.23, \mathrm{p}<0.01)$ and DFA $\alpha 2(\mathrm{r}=0.22, \mathrm{p}<0.01)$ and negatively with DFA $\alpha 1(\mathrm{r}=-0.20, \mathrm{p}<0.02)$. DFA $\alpha 1$ also correlated negatively with subjectively perceived exhaustion from adjectives scale $(r=-0.17, p<0.05)$.

DFA $\alpha 2$ correlated with feeling of stress from adjectives scale $(r$ $=0.20, p<0.02)$ and occurrence of stressful events reported in subjective checklist $(\mathrm{r}=0.17, \mathrm{p}<0.05)$.

\section{Discussion}

Naturalistic design proposed in this study was used to evaluate $\mathrm{HR}$ and HRV changes and subjective feelings of stress during normal day to day life. To make the design the least intrusive to the subjects and to collect reasonable amount of data, we used several repeated measurements, performed at the arranged dates by each subject.

Results from the repeated measurement design reported here showed lowered heart rate (HR) in both allergic and highly anxious subjects. From HRV measures, increases of overall HRV (measured via SDNN, Total power and SD2) as well as increases of HRV on respiratory frequencies (RMSSD, HF, SD1) were observed in both allergy and high trait anxiety group. While these HRV indices associated mostly with parasympathetic influences were increased, similar increases were found also in VLF-HRV, which corresponds to Tran et al. (36), where increases of VLF HRV were interpreted as sympathetic hyperactivation. On the other hand, DFA $\alpha 1$, which is an indicator of fractal attributes of healthy HR complexity, showed lowered values in both allergy and high anxiety group.

\section{Allergy and HRV increase}

Effect of increased HRV was expected in allergy, due to prior research (for a review, see 37). However, the mechanism of ob- served HRV changes in allergy is still not known. One hypothesis states that these effects may be attributed to the role of efferent vagus nerve in anti-inflammatory pathway (38). Increase in vagal activation may be a result of counter regulatory mechanism in response to allergic inflammation (22). On the other hand, HRV increase may also be due to sympathetic withdrawal associated with allergic inflammation itself and Th2 immune response, which increases local sympathetic activity in allergic reaction sites and decreases systemic sympathetic activity $(39,40)$.

\section{$H R V$ changes in high trait anxiety}

While there is more evidence for decrease in HR and increase in HRV for allergy, similar results in the high trait anxiety group are quite surprising. High trait anxiety was in most previous studies associated with an opposite effect of HR increase and HRV decrease (for a review, see 9).

However, findings of dysregulated stress response in highly anxious subjects were reported earlier. Duncko et al. (28) reported blunted cortisol as well as blunted noradrenaline and adrenaline responses, during acute stress in highly trait anxious individuals. These findings were interpreted in context of allostatic model, as inability to react with adaptive stress reaction. While allergy is often associated with increases in anxiety, depression and problems in emotional regulation $(17,18)$, effects of blunted cortisol (41) as well as salivary alpha amylase, which is commonly associated with sympathetic activity (42) and aldosterone (24) were reported for both allergy as well as high trait anxiety.

Based on the mentioned effects we could speculate whether choosing only very high trait anxious subjects (STAI-T score < 49) and contrarily very low anxious subjects as controls (STAI-T <39) could play a role in the observed effects.

While Bornas et al. (26) found differences between high trait anxiety subjects and controls in entropy measures, our results showed no differences in ApEn, however DFA $\alpha 1$ had lower values in both high trait anxiety and allergy group. These are consistent with significant negative correlation of DFA 1 and subjectively perceived stress (via PSS and adjective scales).

\section{Sex differences and between group changes}

Among the sex differences we recorded higher mean HR and lower overall HRV (SDNN, total spectral power, SD2 from Poincaré plot) in women, which is consistent with data from previously done meta-analysis of $172 \mathrm{HRV}$ studies (43). In our data, lower VLF and LF variability in women were observed, while we detected no differences in fast beat-to-beat changes (HF, RMSSD). Differences in non-linear HRV showed higher entropy (ApEn) in women, while DFA 1 was lower consistently with meta-analysis results (43). 
Interestingly, we found sex related differences in HRV changes attributable to allergy and high trait anxiety. All between group effects were more pronounced in women (see Figure 1), while effect of allergy and anxiety in respiratory frequencies HRV (RMSSD, HF, SD1) showed different trends between men and women. Increased vagal HRV parameters were not present in male subjects, though this effect was very strong in women (see Figure 1). These sex differences in vagal HRV related to allergy and anxiety are not clear, and they should be investigated more thoroughly with a larger number of subjects.

Differences between sexes in HRV, with both higher HR and vagally mediated HRV in women while men show higher overall variability, may be related to the effects of estrogen (44), which could attribute to more vagal activation. Moreover, higher levels of oxytocin were also associated with increased vagal tone and slowing of HR (43).

\section{Changes in subjectively perceived stress and $H R V$}

In intergroup comparison, both PSS score and score from stress related adjectives were significantly higher in high trait anxiety group compared to low anxious allergy and control group. An opposite trend was observable in positive affect adjectives, which were lower in high trait anxiety group. Differences between low anxious allergy and control group were small and statistically insignificant for all subjectively experienced stress measures. While stronger reported experience of stress and less positive emotion were expected in high trait anxiety group, an interesting finding is the indifference of allergy group to controls. We assume therefore that differences in subjectively reported stress are related to psychological trait of anxiety, not allergy itself. The association of allergy and higher subjective feelings of stress reported by numerous studies, might be explained by comorbidity of allergy and high trait anxiety described earlier $(17,23)$. Besides intergroup differences, correlation between HRV measures and subjective self-assessment were tested. Correlations were controlled for heart rate, which could partially eliminate strong individual variance in HRV. Positive relationship between VLF-HRV and PSS score was found, indicating higher VLF-HRV in psychological distress. Though VLF-HRV was previously interpreted in relation to sympathetic activity (36), it is also strongly correlated with all other spectral HRV parameters (Total power, LF, HF) indicating both sympathetic and vagal influences.

From non-linear parameters, DFA 1 correlated negatively with perceived stress, while DFA 2 showed opposite tendency. DFA 1 , which is regarded as a marker of short term HRV complexity and healthy HRV was lowered when perceived stress was higher, as well as it was stably lower in allergy and high trait anxiety group. DFA 2 , which should indicate long term complexity, showed exactly opposite manner. Positive correla- tion with PSS score, as well as feelings of stress from adjective scales and reported occurrence of stressful events on the day of the measurement, were reported. DFA 22 is normally correlated with long term variability measures from spectral analysis, VLF and LF HRV (33). Similar trends for VLF-HRV and DFA 22 were apparent in our data as well.

\section{Naturalistic design specifics and limits}

Main aim of the naturalistic design used in this study was to investigate more ecologically valid measurements of stress in follow up to our previously realized laboratory studies. HR, HRV and subjectively experienced stress were measured in response to normal day to day stressors, which were in our data mostly work related $(50 \%)$ or related to personal situations, e.g. relationships etc. $(40.2 \%)$.

Like Bornas et al. (26), we assume that investigating HRV in naturalistic setting may reveal more relevant information on subjects' reactivity during normal functioning. One of the most notable advantages of our design, was very little intrusiveness from the research team and therefore an insight on subject realistic functioning. The use of small and portable eMotion Faros ECG devices enabled us to design this HRV study in the least intrusive way.

On the other hand, there are limitations associated with this kind of design. Even though the cooperation with the subjects was mostly flawless, there were some who due to errors in ECG measurements, movement artifacts or problems in communication had to be excluded from the study, with their data not considered.

Possible confounding variables as movement, use of medication or other substances (caffeine, alcohol etc.) were monitored via self-assessment before each measurement. Possibly, subjects could fail to mention any conditions or medication, which might have been important. Furthermore, possible allergy symptoms in non-allergy group were not tested, only asked about in self-assessment. Therefore, there is a chance of some subjects being allergic despite not knowing about it.

\section{Clinical relevance of the results}

HRV changes in allergy and anxiety described here, as well as relationship between the two might lead to several clinically relevant consequences. Due to similar psychophysiological changes (24) and reported comorbidity of anxiety and allergy $(16,17,18,23)$ as well as the role of stress in allergic process (15), psychological interventions aimed on better coping and reduction of anxiety could be beneficial to allergic patients (45). Similarly, interventions based on normalization of HRV such as HRV biofeedback might also be employed, which has already been demonstrated in a study with asthma (46). Future research is necessary to evaluate 
the effectiveness of different psychological interventions and their contribution to the treatment of allergy.

\section{Conclusion}

This study investigated HRV differences in allergic and highly anxious subjects during their normal day to day functioning. Complete analysis of HRV results showed decreased HR and increased overall HRV in both allergy and high trait anxiety group. While between group differences were most notable in overall HRV indices (SDNN, total spectral power, VLF, SD2) increases in vagal HRV indices (RMSSD, HF-HRV, SD1) were also present. From non-linear HRV indices, lowered DFA 1 in both allergy and high trait anxiety was observed. Interestingly, HRV differences related to allergy and anxiety interacted with subjects' sex showing notable increases in HRV in women, while no significant effects were observed in men.

Subjective measures of stress showed increases in high trait anxiety group, while low-anxious allergy group scored similarly to controls. Based on this, we assume that previously reported increase in subjectively experienced stress in allergy could be a consequence of difference in trait anxiety.

Correlations between HRV indices and subjectively reported feeling of stress were found for PSS and adjective scales and non-linear DFA $\alpha 1$ and $\alpha 2$, showing opposite tendencies.

Research design used in this study offered a naturalistic approach to monitoring of HRV in everyday life stressful situations.

\section{Conflict of interest}

The authors declare that they have no conflict of interest.

\section{Acknowledgements}

This study was supported by grant projects: APVV-0496-12; VEGA 1/0739/17; KEGA 085UK-4/2017; Comenius University grant G-17-021-00, and Nadácia Tatra banky 2016vs029.

\section{References}

1. Ginsberg JP. Editorial: Dysregulation of Autonomic Cardiac Control by Traumatic Stress and Anxiety. Front Psychol 2016; 7. https://doi.org/10.3389/fpsyg.2016.00945.

2. McEwen BS, and Wingfield JC. The concept of allostasis in biology and biomedicine. Horm Behav 2003; 43(1), 2-15. https://doi. org/10.1016/S0018-506X(02)00024-7.

3. McEwen BS. Brain on Stress: how the social environment gets under the skin. P Natl Acad Sci Usa 2012; 109 (Suppl 2):17180-5.

4. 4. Goldberger AL. Non-linear dynamics for clinicians: Chaos theory, fractals, and complexity at the bedside. Lancet 1996; 347(9011):1312-4. doi:10.1016/S0140-6736(96)90948-4.

5. Berntson GG, Thomas Bigger Jr. J, Eckberg DL, Grossman P, Kaufmann PG, Malik M., . . . Van Der Molen MW. Heart rate variability: Origins methods, and interpretive caveats. Psychophysiology 1997; 34(6):623-48.

6. Thayer, J. F., Lane, R. D. A model of neurovisceral integration in emotion regulation and dysregulation. J Affect Disorders 2000; 61;201-16.

7. Thayer JF, Åhs F, Fredrikson M, Sollers JJ, and Wager TD. A meta-analysis of heart rate variability and neuroimaging studies: Implications for heart rate variability as a marker of stress and health. Neurosci Biobehav R 2012; 36(2):747-56. https://doi. org/10.1016/j.neubiorev.2011.11.009.

8. Spielberger CD, Gorsuch, RL, Lushene PR, Vagg PR, and Jacobs AG (Eds.). Manual for the state-trait anxiety inventory (form Y). Palo Alto: Consulting Psychologists Press, Inc 1983.

9. Friedman BH. (2007). An autonomic flexibility-neurovisceral integration model of anxiety and cardiac vagal tone. Biol Psychol 1983; 74(2):185-99. https://doi.org/10.1016/j.biopsycho.2005.08.009.

10. Rozanski A, Blumenthal JA, and Kaplan J. Impact of Psychological Factors on the Pathogenesis of Cardiovascular Disease and Implications for Therapy. Circulation 1999; 99:2192-217.

11. Chalmers JA, Quintana DS, Abbott M. JA, and Kemp AH. Anxiety Disorders are associated with reduced heart rate variability: A meta-analysis. Front Psychiatry 2014; 5. https://doi.org/10.3389/ fpsyt.2014.00080.

12. Fuller BF. The effects of stress-anxiety and coping styles on heart rate variability. Int J Psychophysiol 1992; 12(1):81-6. doi:10.1016/0167-8760(92)90045-D.

13. Miu AC, Heilman RM, and Miclea M. Reduced heart rate variability and vagal tone in anxiety: Trait versus state, and the effects of autogenic training. Auton Neurosci 2009; 145(1-2):99-103. https://doi.org/10.1016/j.autneu.2008.11.010.

14. Dishman RK, Nakamura Y, Garcia ME, Thompson RW, Dunn $\mathrm{AL}$, and Blair SN. Heart rate variability, trait anxiety, and perceived stress among physically fit men and women. Int J Psychophysiol 2000;37(2):121-33.

15. Suárez AL, Feramisco JD, Koo J, and Steinhoff M. Psychoneuroimmunology of psychological stress and atopic dermatitis: Pathophysiologic and therapeutic updates. Acta Derm-Venereol 2012; 92(1):7-15. doi:10.2340/00015555-1188.

16. Hashizume H, Horibe T, Ohshima A, Ito T, Yagi H, and Takigawa M. Anxiety accelerates T-helper 2-tilted immune responses in patients with atopic dermatitis. Br J Dermatol 2005; 152(6):1161-4. https://doi.org/10.1111/j.1365-2133.2005.06449.

17. Hashizume $\mathrm{H}$, and Takigawa M. Anxiety in allergy and atopic dermatitis. Curr Opin Allergy Clin Immunol 2006; 6(5):335-9. doi:10.1097/01.all.0000244793.03239.40.

18. Yamamoto Y, Yamazaki S, Hayashino Y, Takahashi O, Tokuda Y, Shimbo T, . . . Fukuhara S. Association between frequency of pruritic symptoms and perceived psychological stress: A Japanese population-based study. Arch Derm Syph 2009;145(12): 1384-8. doi:10.1001/archdermatol.2009.290.

19. Yokusoglu M, Ozturk S, Uzun M, Baysan O, Demirkol S, Caliskaner Z, ... Isik E. Heart rate variability in patients with allergic rhinitis. Mil Med 2007; 172(1):98-101.

20. Lan M, Lee G, Shiao A, Ko J, and Shu C. Heart Rate Variability Analysis in Patients with Allergic Rhinitis. Sci World J 2013; $1-5$.

21. Tascilar E, Yokusoglu M, Dundaroz R, Baysan O, Ozturk S, Yozgat $\mathrm{Y}$, and Kilic A. Cardiac Autonomic Imbalance in Children with Al- 
lergic Rhinitis. Tohoku J Exp Med 2009; 219(3):187-91. https:// doi.org/10.1620/tjem.219.187.

22. Boettger MK, Bär KJ, Dohrmann A, Müller H, Mertins L, Brockmeyer NH, and Agelink MW. Increased vagal modulation in atopic dermatitis. J Dermatol Sci 2009; 53(1):55-9. https://doi. org/10.1016/j.jdermsci.2008.08.003.

23. Solarikova P, Brezina I, Turonova D, Rajcani J. Anxiety and sympathetic response to stress in allergic patients. Activ Nerv Super Rediviva 2016; 58:88-94.

24. Hlavacova N, Solarikova P, Marko M, Brezina I, Jezova D. Blunted cortisol response to psychosocial stress in atopic patients is associated with decrease in salivary alpha-amylase and aldosterone: Focus on sex and menstrual cycle phase. Psychoneuroendocrino 2017; 78:31-8.

25. Schlotz W, Schulz P, Hellhammer J, Stone AA, and Hellhammer $\mathrm{DH}$. Trait anxiety moderates the impact of performance pressure on salivary cortisol in everyday life. Psychoneuroendocrino 2006; 31(4):459-72. http://doi.org/10.1016/j.psyneuen.2005.11.003.

26. Bornas X, Balle M, De la Torre-Luque A, Fiol-Veny A, and Llabrés J. Ecological assessment of heart rate complexity: Differences between high- and low-anxious adolescents. Int J Psychophysiol 2015; 98(1):112-8. https://doi.org/10.1016/j.ijpsycho.2015.07.007.

27. Müllner J, Ruisel I, and Farkaš G. Príručka pre administráciu, interpretáciu a vyhodnocovanie dotazníka na meranie úzkosti a úzkostlivosti. Bratislava: Psychodiagnostické a didaktické testy. 1980; 93.

28. Duncko R, Makatsori A, Fickova E, Selko D, and Jezova D. Altered coordination of the neuroendocrine response during psychosocial stress in subjects with high trait anxiety. Prog Neuro-Psychoph 2006; 30(6):1058-66. https://doi.org/10.1016/j. pnpbp.2006.04.002.

29. Cohen S, Kamarck T, and Mermelstein R. A global measure of perceived stress. J Health Soc Behav 1983; 385-96.

30. Tarvainen MP, Niskanen JP, Lipponen JA, Ranta-aho PO, and Karjalainen PA. Kubios HRV - Heart rate variability analysis software. Comp Meth Programs Biomed 2014; 113(1):210-20.

31. Task Force of the European Society of Cardiology and the North American Society of Pacing and Electrophysiology. Heart rate variability standards of measurement, physiological interpretation, and clinical use. Eur Heart J 1996; 17(3):354-81.

32. Billman GE. The LF/HF ratio does not accurately measure cardiac sympatho-vagal balance. Front Physiol 2013; 4. https://doi. org/10.3389/fphys.2013.00026.

33. Voss A, Schulz S, Schroeder R, Baumert M, and Caminal P. Methods derived from nonlinear dynamics for analysing heart rate variability. Philos T Roy Soc A 2009; 367(1887):277-96. https://doi. org/10.1098/rsta.2008.0232.

34. Peng CK, Havlin S, Stanley HE, and Goldberger AL. Quantification of scaling exponents and crossover phenomena in nonstationary heartbeat time series. Chaos 1995; 5: 82-7. doi:10.1063/1.166141.
35. Blackwell E, Carlos F, Mendes De Leon F, Miller G. Applying mixed regression models to the analysis of repeated - measures data in psychosomatic medicine. Psychosom Med 2006; 68:870-8

36. Tran B, Papoiu A, Russoniello C, Wang H, Patel T, Yosipovitch G, and Chan Y. Effect of Itch, Scratching and Mental Stress on Autonomic Nervous System Function in Atopic Dermatitis. Acta Derm-Venereol 2010; 90(4):354-61. https://doi. org/10.2340/00015555-0890.

37. De Abreu LC, Vilar de Assis E, De Andrade Isidório U, Do Nascimento Andrade Feitosa A, Nunes Alves de Sousa M, Bezerra de Menezes Silveira G, et al. Autonomic Nervous System and Allergic Diseases: Integrative Literature Review. Int Arch Med 2015; 8:1-6.

38. Huston JM, and Tracey KJ. The pulse of inflammation: heart rate variability, the cholinergic anti-inflammatory pathway and implications for therap. Symposium: The pulse of inflammation: implications for therapy. J Intern Med 2011; 269(1):45-53. https://doi. org/10.1111/j.1365-2796.2010.02321.

39. Shahabi S, Hassan ZM, Jazani NH, and Ebtekar M. Sympathetic nervous system plays an important role in the relationship between immune mediated diseases. Med Hypotheses 2006; 67(4):900-3. https://doi.org/10.1016/j.mehy.2006.03.039.

40. Elenkov IJ, Wilder RL, Chrousos GP, and Vizi ES. The sympathetic nerve - an integrative interface between two supersystems: the brain and the immune system. Pharmacol Rev 2000; 52(4):595-638.

41. Buske-Kirschbaum A, Geiben A, Höllig H, Morschhäuser E, and Hellhammer D. Altered responsiveness of the hypothalamus-pituitary-adrenal axis and the sympathetic adrenomedullary system to stress in patients with atopic dermatitis. J Clin Endocr Metab 2002; 87(9):4245-51. https://doi.org/10.1210/ jc.2001-010872.

42. Nater UM, and Rohleder N. Salivary alpha-amylase as a non-invasive biomarker for the sympathetic nervous system: Current state of research. Psychoneuroendocrino 2009; 34(4):486-96. https:// doi.org/10.1016/j.psyneuen.2009.01.014.

43. Koenig J, and Thayer JF. Sex differences in healthy human heart rate variability: A meta-analysis. Neurosc Biobehav R 2016; 64:288-310. http://doi.org/10.1016/j.neubiorev.2016.03.007.

44. Du XJ, Fang L, Kiriazis H. Sex dimorphism in cardiac pathophysiology: experimental findings, hormonal mechanisms, and molecular mechanisms. Pharmacol Ther 2006; 111:434-75.

45. Chida Y, Steptoe A, Hirakawa N, Sudo N, and Kubo C. The Effects of Psychological Intervention on Atopic Dermatitis. Int Arch Allergy Immunol 2007; 144(1):1-9. https://doi. org/10.1159/000101940.

46. Lehrer PM, Vaschillo E, Vaschillo B, Lu SE, Scardella A, Siddique M, Habib R. Biofeedback treatment for asthma. Chest Journal 2004; 126(2):352-61. 